\title{
Efeito de Trilhas sobre Artrópodes de Serrapilheira no Parque Nacional da Tijuca, RJ
}

\author{
Felipe Drummond-Alves ${ }^{1}$, Rafaela Dias Antonini ${ }^{1}$, André Felippe Nunes-Freitas ${ }^{1}$ \\ ${ }^{1}$ Departamento de Ciências Ambientais, Universidade Federal Rural do Rio de Janeiro - UFRRJ, Seropédica/RJ, Brasil
}

\begin{abstract}
RESUMO
Foi analisado o efeito de trilhas sobre a comunidade de artrópodes de serrapilheira em duas trilhas do Parque Nacional da Tijuca. Amostras de serrapilheira foram coletadas em duas cotas altitudinais ( 650 e $850 \mathrm{~m}$ anm) e em três classes de distância $(0,5$ e $10 \mathrm{~m}$ ). Os artrópodes foram identificados ao nível de ordem. Foram amostrados 4.491 indivíduos de 19 taxa, sendo Orthoptera a mais frequente. Foram encontradas diferenças significativas no número de táxons entre trilhas, possivelmente como resposta a um efeito causal da estrutura e da composição florística das trilhas e das cotas. A trilha que apresentou os maiores valores de riqueza foi a do Bico do Papagaio, diferindo da trilha do Pico da Tijuca. Concluiu-se que as diferenças encontradas podem estar relacionadas com o grau de perturbação diferenciado que as duas trilhas sofrem. No entanto, análises mais detalhadas poderão tornar os resultados mais claros.
\end{abstract}

Palavras-chave: Floresta Atlântica, cotas altitudinais, uso público.

\section{Effect of Trails on Litter Arthropods in the Tijuca National Park, Brazil}

\begin{abstract}
The effect of trails on litter arthropod community in two tracks of the Tijuca National Park was analyzed. Samples of litter were collected at two altitudes (650 and 850 m.a.s.l.) and three distance classes $(0,5$ and $10 \mathrm{~m})$. Arthropods were identified on order level. There were 4491 sampled individuals of 19 taxa, and Orthoptera was the most frequent. There were significant differences in the number of taxon between tracks, possibly as a response to a causal effect of the structure and floristic composition of trails and quotes. The trail that showed the highest richness levels was Bico do Papagaio, differing from Pico da Tijuca. It is suggested that the differences may be related to the degree of disturbance that differentiated the two tracks. However, more detailed analysis can make clearer results.
\end{abstract}

Keywords: Atlantic rainforest, elevation, public use.

\section{INTRODUÇÃO}

A Floresta Atlântica apresenta uma grande variedade de formações, englobando um diversificado conjunto de ecossistemas florestais com estruturas e composições florísticas bastante diferenciadas (Oliveira-Filho \& Fontes, 2000). Em geral, as diferenças entre localidades, na estrutura e na composição florística, estão relacionadas com as variações climáticas entre as regiões de ocorrência, tendo como elemento comum a exposição aos ventos úmidos que sopram do oceano (Oliveira-Filho \& Fontes, 2000).

Mesmo estando reduzida a uma pequena parte da sua extensão original, a Floresta Atlântica está incluída 
entre um dos 34 hotspots mundiais, que são áreas com extraordinária diversidade e elevada concentração de espécies endêmicas, a qual vem sofrendo elevada e rápida perda de hábitat (Myers et al., 2000; Mittermeier et al., 2005).

Segundo projeções históricas, o Estado do Rio de Janeiro apresentava cerca de $97 \%$ da sua área recoberta por Floresta Atlântica, o equivalente a aproximadamente 44 mil km² de florestas (Fundação SOS Mata Atlântica, 1993; Rocha et al., 2003). Atualmente, a ampla área de floresta que cobria o estado se resume a numerosas manchas florestais, encontradas principalmente nas vertentes das cadeias montanhosas, acima de $500 \mathrm{~m}$ acima do nível médio do mar (anm) (Tanizaki-Fonseca \& Moulton, 2000; Fundação SOS Mata Atlântica, 2002; Rocha et al., 2003). Esses fragmentos somam cerca de $8 \mathrm{mil} \mathrm{km}^{2}$, o equivalente a $17 \%$ da área original (Tanizaki-Fonseca \& Moulton, 2000). O Rio de Janeiro tem uma fundamental importância na conservação da Floresta Atlântica por apresentar grandes blocos de vegetação contínua com elevado grau de conectividade (Rocha et al., 2003). Da área remanescente de florestas do estado, cerca de 50\% está protegida em unidades de conservação de diferentes tipos (Tanizaki-Fonseca \& Moulton, 2000; Fundação SOS Mata Atlântica, 2002), sendo os parques nacionais as mais importantes.

As funções dos parques nacionais são variadas, sendo uma delas a recreação em contato com a natureza, o turismo ecológico (Brasil, 2000), que se realizada de forma sustentável, incentiva a conservação e a formação de uma consciência ambientalista, promovendo o bem-estar dos atores envolvidos (Zacchi, 2004). Segundo Alves \& Dias (2005), os praticantes do turismo ecológico possuem uma preocupação ecológica diferente dos praticantes de outras atividades esportivas, pois ela envolve uma forte sinergia entre a prática esportiva e a natureza. No entanto, a prática desses esportes pode causar um intenso impacto sobre o ambiente dentro das unidades de conservação, em especial nos locais onde há maior taxa de visitação, já que, em geral, tende a utilizar com maior intensidade áreas específicas, como as trilhas.

Estudos têm demonstrado que as comunidades edáficas sofrem uma ação rápida e direta das perturbações, modificando a abundância, a riqueza e a composição de grupos indicadores que dependem de certos recursos do sistema para manter populações estáveis (Garay \& Nataf, 1982; Silveira et al., 1995; Pellens \& Garay, 1999a, b) os quais podem funcionar, inclusive, como indicadores de degradação (Garay \& Nataf, 1982; Hafidi et al., 1998; Mcgeoch, 1998; Pellens \& Garay, 1999a, b; Wink et al., 2005; Freitas et al., 2006a). Como as respostas dessas comunidades aos diferentes tipos de ação antrópica são rápidas, qualquer tipo de alteração do hábitat leva a modificações relacionadas a perdas e alteração da riqueza, da composição e da diversidade (Correia \& Oliveira, 2000). No entanto, são poucos os estudos sobre o efeito da ação antrópica causada pela visitação sobre a comunidade de artrópodes edáficos (e.g., Garay \& Nataf, 1982).

Dessa forma, este estudo visa avaliar se trilhas de visitação turística modificam a estrutura, a riqueza e a composição da comunidade de artrópodes de serrapilheira no Parque Nacional da Tijuca, Rio de Janeiro, RJ, buscando responder as seguintes perguntas: i) Qual a diversidade e a abundância das comunidades de macroartrópodes de serrapilheira em trilhas com taxas de visitação e, consequentemente, graus de impactos distintos?; ii) Existem diferenças na composição das comunidades de macroartrópodes de serrapilheira em diferentes altitudes?; iii) Qual a relação das possíveis diferenças na composição das comunidades com o grau de visitação das trilhas?

\section{MATERIAL E MÉTODOS}

\section{1. Área de estudo}

O estudo foi realizado em duas trilhas do Parque Nacional da Tijuca (PNT), localizadas nas coordenadas geográficas $22^{\circ} 55^{\prime}$ e $23^{\circ} 01^{\prime} \mathrm{S}$ e $43^{\circ} 12^{\prime}$ e $43^{\circ} 19^{\prime} \mathrm{W}$. O PNT é uma das maiores florestas urbanas do mundo, apresentado uma área total de 3. 200 ha, dividida em três grandes subunidades: a Floresta da Tijuca (onde foi realizado o estudo), a Serra da Carioca e a Pedra da Gávea/Pedra Bonita (Freitas et al., 2006b). O PNT é um dos principais remanescentes de Floresta Atlântica do Estado do Rio de Janeiro (Rocha et al., 2003), possuindo elevado grau de importância para a conservação da flora e da fauna do município e do estado (Freitas et al., 2006b).

O clima na região é tropical, com verões úmidos e invernos secos (tipo Aw de Köppen-Geiger). A temperatura média anual que varia de $22^{\circ} \mathrm{C}$ e $24^{\circ} \mathrm{C}$, apresentando máxima absoluta de $38^{\circ} \mathrm{C}$ a $40^{\circ} \mathrm{C}$ nos 
meses de dezembro a fevereiro, e mínimas de $4{ }^{\circ} \mathrm{C}$ a $8^{\circ} \mathrm{C}$ nos meses de junho e julho. A pluviosidade anual varia de $1.250 \mathrm{~mm}$ a $1.500 \mathrm{~mm}$ (Matos et al., 2002), podendo chegar a $2.000 \mathrm{~mm}$ nos anos de maior pluviosidade (Freitas et al., 2006b).

Para o presente estudo foram selecionadas duas das trilhas de maior visitação no PNT: a trilha do Pico da Tijuca (PT; $22^{\circ} 56^{\prime} 30,08^{\prime \prime}$ 'S; $42^{\circ} 17^{\prime} 15,26^{\prime \prime}$ O) e a do Bico do Papagaio (BP; $22^{\circ} 56^{\prime} 43,70^{\prime \prime}$ S; $43^{\circ} 19^{\prime} 4,07^{\prime \prime} \mathrm{O}$ ). As duas trilhas apresentam características semelhantes quanto a sua cobertura vegetal, composta basicamente por vegetação secundária em avançado estado de regeneração, entremeada com trechos de vegetação baixa, formada por diferentes espécies arbustivas e herbáceas. A trilha PT possui uma extensão de 2,5 km em terreno acidentado e, muitas vezes, íngreme, com subida constante sem muita variação de inclinação, sendo considerada leve. Já a trilha BP é considerada de leve a moderada, de curta duração, apresentando a mesma extensão da PT. Segundo informação coletada com os agentes do PNT, as trilhas apresentam taxas de visitação distintas, sendo a do Pico da Tijuca mais visitada do que a do Bico de Papagaio (Parque Nacional da Tijuca, dados não publicados).

\subsection{Metodologia}

Para avaliar o efeito das trilhas sobre as comunidades de artrópodes de serapilheira (Correia \& Oliveira, 2000), foram utilizadas parcelas de $625 \mathrm{~cm}^{2}$ (Hafidi et al., 1998; Pellens \& Garay, 1999a, b), que foram demarcadas com auxílio de um gabarito de madeira com laterais de $25 \mathrm{~cm} \times 25 \mathrm{~cm}$. As coletas ocorreram no pico da estação chuvosa de 2006/2007 (segunda quinzena de janeiro) e da estação seca (entre o final do mês de junho e início julho de 2007). Em cada uma das trilhas foram coletadas 6 amostras, sendo 3 amostras na altitude de $650 \mathrm{~m}$ anm e 3 amostras na altitude $820 \mathrm{~m}$ anm, as quais totalizaram 24 amostras ao final do período de estudo. Em cada estação estudada, as amostras foram coletadas ao longo de três dias consecutivos. As parcelas foram alocadas a distâncias de $0 \mathrm{~m}, 5 \mathrm{~m}$ e $10 \mathrm{~m}$ da borda de cada trilha. Para reduzir a dependência entre as amostras, a alocação das parcelas foi sorteada tanto para a distância quanto para sua localização em relação à trilha (lado esquerdo ou direito), em parcelas não fixas. Após o sorteio e a demarcação, toda a serrapilheira presente dentro da parcela foi coletada, acondicionada e lacrada em sacos plásticos de $20 \mathrm{~L}$, de forma a evitar a perda de indivíduos e para posterior triagem no laboratório.

Em laboratório, toda a serrapilheira coletada foi colocada em funis de Berlese (Hafidi et al., 1998; Pellens \& Garay, 1999a, b), onde permaneceu por cinco dias. Após esse período, todo o material coletado nos frascos foi triado e identificado ao nível ordem, de acordo com Buzzi (2002) e Triplehorn \& Johnson (2011), sendo considerados, na triagem, apenas os artrópodes com tamanho variando entre $2 \mathrm{~mm}$ e $20 \mathrm{~mm}$ de comprimento. A composição de cada amostra, baseada no número de morfoespécies, foi listada e, posteriormente, foram calculadas a riqueza (S), a abundância (N) e a frequência (\%) de cada ordem para cada uma das distâncias, altitude e trilha amostrada (Magurran, 1988).

A normalidade dos dados de riqueza e abundância foi calculada através do teste de normalidade de Lilliefors (Zar, 1999), para os dados que não apresentaram distribuição normal, realizou-se a transformação para o logaritmo do valor $(\log x+1)(Z a r, 1999)$. Após essa etapa foram testadas possíveis diferenças entre distâncias, altitudes e trilhas através de análise de variância (ANOVA) (Zar, 1999). Para essa análise, a estação do ano (seca ou chuvosa) foi inserida no modelo matemático da ANOVA como covariável categórica, de forma a se retirar o efeito da estação na análise, já que poderia levar a um erro na interpretação dos resultados (Legendre \& Legendre, 1998).

Para testarmos do grau de similaridade entre trilhas, cotas e distâncias, utilizou-se o método de escalonamento multidimensional não métrico (NMDS). Foi utilizado como medida de distância a distância Jaccard ( $\mathrm{Dj}=1$ - J) (Legendre \& Legendre, 1998). Posteriormente, para verificar se os agrupamentos obtidos diferiram quanto ao seu ranqueamento na ordenação, utilizou-se análise de variância (ANOVA), seguida do teste de Tukey (Zar, 1999). O nível de significância utilizado no estudo foi de $5 \%$.

\section{RESULTADOS}

Nas coletas de verão foram amostrados um total de 3.380 indivíduos de 20 taxa da fauna de macroartrópodes de serrapilheira. Dentre esses, os mais 
abundantes foram Orthoptera $(\mathrm{N}=2.447 ; 72,40 \%) \mathrm{e}$ Formicidae ( $\mathrm{N}=353 ; 10,44 \%)$, enquanto os menos representativos foram Diplopoda, Neuroptera e Dermaptera ( $\mathrm{N}=1$ cada; $0,03 \%$ ) (Tabela 1 ). Já nas coletas de inverno foram amostrados 1.112 indivíduos de 14 diferentes taxa da fauna de macroartrópodes de serrapilheira. Dentre esses, os mais abundantes foram os taxa Orthoptera ( $\mathrm{N}=369 ; 33,13 \%)$ e Formicidae $(\mathrm{N}=295 ; 26,53 \%)$, enquanto os menos representativos foram Dermaptera e Collembola ( $\mathrm{N}=1$ cada; $0,09 \%)$ (Tabela 2). Apesar disso, em nenhum dos modelos testados a estação do ano teve influência sobre a riqueza ou a abundância de indivíduos ( $\mathrm{p}>0,005)$.

A abundância da comunidade de macroartrópodes edáficos variou entre as trilhas, cotas altitudes e distâncias. A trilha PT apresentou maior abundância de indivíduos $(\mathrm{N}=2.636)$, quando comparada com a trilha $\mathrm{BP}(\mathrm{N}=1.856)$, mas elas não diferiram para esse parâmetro $\left(D F=1 ; f=3,634 ; r^{2}=0,269 ; p=0,06\right)$ (Figura 1A). Já a riqueza de espécies foi maior na BP
( $S=19$ famílias), sendo significativa a riqueza da trilha do Pico da Tijuca $\left(\mathrm{DF}=1 ; \mathrm{f}=7,379 ; \mathrm{r}^{2}=0,079\right.$; $\mathrm{p}=0,008)$ (Figura 1B).

Na PT foi observada uma maior abundância de macroartrópodes na altitude de $850 \mathrm{~m}(\mathrm{~N}=1.632)$, quando comparada a altitude de $650 \mathrm{~m}(\mathrm{~N}=1.004)$, porém não se observou diferença significativa para essa variável quando comparadas as duas altitudes $\left(\mathrm{DF}=1 ; \mathrm{f}=3,387 ; \mathrm{r}^{2}=0,369 ; \mathrm{p}=0,072\right.$ ) (Figura 2A). Quando se avaliaram os valores de riqueza, observou-se que os maiores valores foram encontrados na cota de $650 \mathrm{~m}(\mathrm{~S}=13)$, havendo diferença significativa entre as duas classes de altitude da trilha $(\mathrm{DF}=1 ; \mathrm{f}=3,869$; $\mathrm{r}^{2}=0,086 ; \mathrm{p}=0,050$ ) (Figura 2C). Já na BP, a altitude de $650 \mathrm{~m}$ apresentou maior abundância $(\mathrm{N}=1.256)$, havendo diferença significativa entre as duas cotas $\left(\mathrm{DF}=1 ; \mathrm{f}=15,323 ; \mathrm{r}^{2}=0,398 ; \mathrm{p}<0,001\right)$ (Figura 2B). Os maiores valores de riqueza também foram registrados na cota de $650 \mathrm{~m}(\mathrm{~S}=17)$, que diferiu da cota $850 \mathrm{~m}$ $\left(\mathrm{DF}=1 ; \mathrm{f}=40,005 ; \mathrm{r}^{2}=0,473 ; \mathrm{p}<0,001\right)($ Figura $2 \mathrm{D})$.

Tabela 1. Composição e abundância da comunidade de artrópodes nas diferentes distâncias, em duas altitudes, nas trilhas do Bico do Papagaio e do Pico da Tijuca, no verão, Parque Nacional da Tijuca, Rio de Janeiro, Brasil.

Table 1. Composition and abundance of arthropod community at different distances in two elevations of Bico do Papagaio and Pico da Tijuca trails, in the summer, Tijuca National Park, Rio de Janeiro, Brazil.

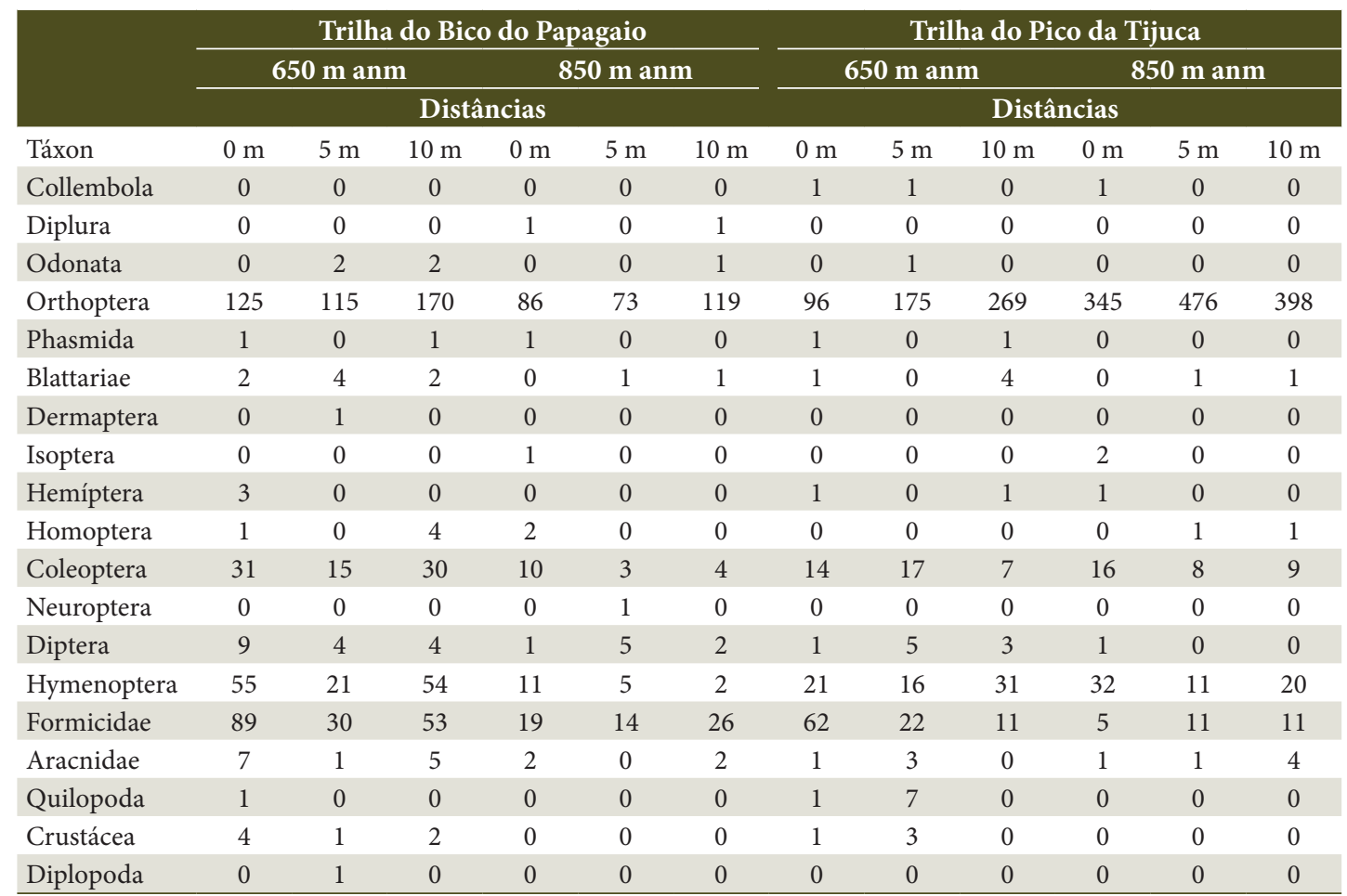


Tabela 2. Composição e abundância da comunidade de macroartrópodes de serrapilheira nas diferentes distâncias, em duas altitudes, nas trilhas do Bico do Papagaio e do Pico da Tijuca, no inverno, no Parque Nacional da Tijuca, Rio de Janeiro, Brasil.

Table 2. Composition and abundance of arthropod community at different distances of two altitude of Bico do Papagaio and Pico da Tijuca trails, in the winter, Tijuca National Park, Rio de Janeiro, Brazil.

\begin{tabular}{|c|c|c|c|c|c|c|c|c|c|c|c|c|}
\hline & \multicolumn{6}{|c|}{ Trilha do Bico do Papagaio } & \multicolumn{6}{|c|}{ Trilha do Pico da Tijuca } \\
\hline & \multicolumn{3}{|c|}{$650 \mathrm{~m}$ anm } & \multicolumn{3}{|c|}{$850 \mathrm{~m}$ anm } & \multicolumn{3}{|c|}{$650 \mathrm{~m}$ anm } & \multicolumn{3}{|c|}{$850 \mathrm{~m}$ anm } \\
\hline & \multicolumn{6}{|c|}{ Distâncias } & \multicolumn{6}{|c|}{ Distâncias } \\
\hline Táxon & $0 \mathrm{~m}$ & $5 \mathrm{~m}$ & $10 \mathrm{~m}$ & $0 \mathrm{~m}$ & $5 \mathrm{~m}$ & $10 \mathrm{~m}$ & $0 \mathrm{~m}$ & $5 \mathrm{~m}$ & $10 \mathrm{~m}$ & $0 \mathrm{~m}$ & $5 \mathrm{~m}$ & $10 \mathrm{~m}$ \\
\hline Collembola & 0 & 1 & 0 & 0 & 0 & 0 & 0 & 0 & 0 & 0 & 0 & 0 \\
\hline Diplura & 0 & 0 & 0 & 0 & 0 & 0 & 0 & 0 & 0 & 0 & 0 & 0 \\
\hline Odonata & 0 & 0 & 0 & 0 & 0 & 0 & 0 & 0 & 0 & 0 & 0 & 0 \\
\hline Orthoptera & 16 & 30 & 26 & 31 & 25 & 46 & 6 & 25 & 30 & 43 & 38 & 53 \\
\hline Phasmida & 0 & 0 & 1 & 0 & 0 & 1 & 1 & 1 & 0 & 0 & 0 & 1 \\
\hline Blattariae & 1 & 3 & 3 & 1 & 0 & 1 & 1 & 0 & 3 & 0 & 0 & 0 \\
\hline Dermaptera & 0 & 1 & 0 & 0 & 0 & 0 & 0 & 0 & 0 & 0 & 0 & 0 \\
\hline Isoptera & 4 & 2 & 3 & 1 & 5 & 7 & 0 & 0 & 0 & 2 & 0 & 3 \\
\hline Hemíptera & 1 & 0 & 0 & 0 & 0 & 0 & 0 & 0 & 1 & 2 & 0 & 0 \\
\hline Homoptera & 1 & 0 & 5 & 1 & 0 & 0 & 0 & 0 & 0 & 1 & 1 & 1 \\
\hline Coleoptera & 21 & 4 & 24 & 3 & 1 & 3 & 4 & 5 & 5 & 8 & 4 & 5 \\
\hline Neuroptera & 0 & 0 & 0 & 0 & 0 & 0 & 0 & 0 & 0 & 0 & 0 & 0 \\
\hline Diptera & 8 & 5 & 7 & 0 & 5 & 4 & 2 & 7 & 2 & 1 & 1 & 1 \\
\hline Hymenoptera & 29 & 8 & 33 & 7 & 2 & 1 & 30 & 7 & 28 & 23 & 13 & 26 \\
\hline Formicidae & 53 & 28 & 62 & 8 & 13 & 32 & 37 & 15 & 5 & 2 & 18 & 22 \\
\hline Aracnidae & 3 & 5 & 10 & 1 & 5 & 2 & 2 & 2 & 0 & 0 & 1 & 5 \\
\hline Quilopoda & 0 & 0 & 0 & 0 & 0 & 0 & 0 & 2 & 1 & 0 & 0 & 0 \\
\hline Crustácea & 4 & 1 & 2 & 0 & 0 & 0 & 1 & 3 & 0 & 0 & 0 & 0 \\
\hline Diplopoda & 0 & 0 & 0 & 0 & 0 & 0 & 0 & 0 & 0 & 0 & 0 & 0 \\
\hline
\end{tabular}

No PT, a distância de $10 \mathrm{~m}$ foi a que apresentou maior número de indivíduos $(\mathrm{N}=963)$, enquanto a classe de distância $0 \mathrm{~m}$ apresentou menor abundância $(\mathrm{N}=771)$ (Figura $2 \mathrm{~A})$. No entanto, as três classes de distância não diferiram significativa $\left(\mathrm{DF}=2 ; \mathrm{f}=0,228 ; \mathrm{r}^{2}=0,328 ; \mathrm{p}=0,797\right)$ (Figura 3A). As diferentes distâncias estudadas também não diferiram significativamente em relação ao número de morfoespécies amostradas $(\mathrm{DF}=2 ; \mathrm{f}=1,184$; $\mathrm{r}^{2}=0,058 ; \mathrm{p}=0,316$ ) (Figura $3 \mathrm{C}$ ). Já no BP, a classe de distância com maior número de indivíduos foi a de $10 \mathrm{~m}(\mathrm{~N}=758)$, enquanto a com menor abundância foi a de $5 \mathrm{~m}(\mathrm{~N}=442)$. No entanto, não houve diferenças significativas entre as três classes de distância $\left(\mathrm{DF}=1 ; \mathrm{f}=2,206 ; \mathrm{r}^{2}=0,266 ; \mathrm{p}=0,122\right.$ ) (Figura 3B). Também não houve diferença significativa entre a riqueza das três classes $\left(\mathrm{DF}=1 ; \mathrm{f}=0,192 ; \mathrm{r}^{2}=0,014\right.$; $\mathrm{p}=0,826)$ (Figura 3D).

A análise de ordenação (NMDS) agrupou as cotas altitudinais inferiores $(650 \mathrm{~m})$ independentemente da trilha estudada (Figura 4), havendo diferenças no ranqueamento das áreas em relação ao eixo 1 do $\operatorname{NMDS}\left(\mathrm{DF}=1 ; \mathrm{f}=7,181 ; \mathrm{r}^{2}=0,646 ; \mathrm{p}=0,023\right)$, mas não em relação ao eixo $2(\mathrm{p}=0,618)$.

\section{DISCUSSÃO}

Os dados sobre a composição da comunidade mostraram que os dois táxons mais abundantes foram a ordem Orthoptera e a família Formicidae (Ordem Hymenoptera), respectivamente. No entanto, estudos indicam que a ordem Orthoptera normalmente é considerada rara na composição da comunidade de macroartrópodes de serrapilheira (Pellens \& Garay, 1999a, b). Algumas espécies de ortópteros apresentam um ciclo de vida homodinâmico, no qual ocorre recrutamento de novos indivíduos ao longo de todo o ano, com pequenas oscilações na população (Masaki \& Walker, 1987). No entanto, alguns Orthopteras apresentam explosão populacional, com grande número de indivíduos 

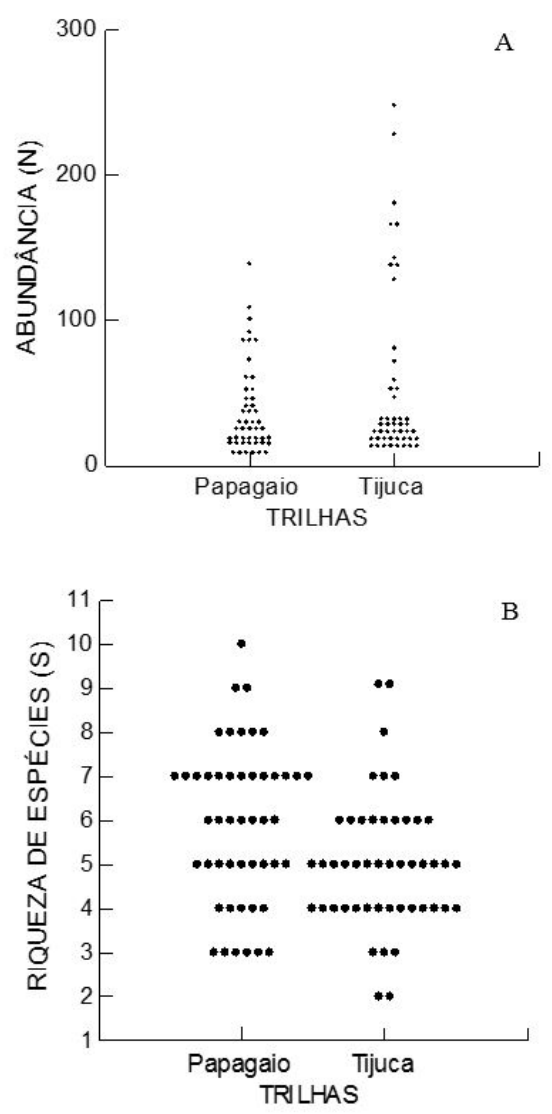

Figura 1. Abundância de indivíduos (A) e riqueza de espécies (B) da comunidade de artrópodes de serrapilheira amostrados nas trilhas do Bico do Papagaio (Papagaio) e do Pico da Tijuca (Tijuca), no Parque Nacional da Tijuca, Rio de Janeiro, Brasil.

Figure 1. Individual abundance (A) and species richeness (B) of arthropods community sampled at Bico do Papagaio and Pico da Tijuca trails, Tijuca National Park, Rio de Janeiro, Brazil.

sendo recrutados em um mesmo momento do ano, especialmente nos períodos de maior produtividade (verão) (Lecoq, 1991). Isso também explicaria a elevada proporção desse grupo no período chuvoso, período de maior produtividade de artrópodes devido às condições climáticas mais favoráveis e a maior disponibilidade de recursos (Janzen, 1973; Wolda, 1977, 1978; Denlinger, 1980; Wolda \& Fisk, 1981). Dessa forma, é possível que a elevada abundância esteja relacionada a esses fatores, especialmente pelo fato de esses organismos apresentarem um hábito alimentar generalista, podendo consumir desde outros animais até plantas (Buzzi, 2002).
Já o segundo grupo mais abundante (Formicidae), apesar de não ser o mais abundante entre os artrópodes, constitui 15\% da biomassa total de insetos nas florestas tropicais (Freitas et al., 2006a), especialmente por sua forma de vida social (Pellens \& Garay, 1999a, b). Estudos têm demonstrado que tanto a riqueza quanto a abundância e a biomassa de espécies de formigas podem ser alteradas por um conjunto de fatores, em especial aqueles associados a alterações na estrutura da vegetação (Freitas et al., 2006a). Dessa forma, é possível que os valores encontrados para esse grupo sejam um efeito causal secundário do efeito das trilhas, já que estas alterariam a estrutura da vegetação e, consequentemente, a densidade e biomassa desse grupo.

Quando analisados separadamente, os dados de número de táxons por trilha, cota altitudinal e classe de distância, observou-se que os padrões de abundância e frequência são mantidos, com algumas alterações na composição, especialmente quando considerados os táxons mais raros. Apesar de essa mudança na composição de espécies ser um padrão esperado, especialmente em termos altitudinais (Janzen, 1973; Rosenzweig, 2000), a pouca variação pode dever-se ao nível de identificação, que chegou apenas até o nível de ordem na maioria dos grupos. Possivelmente, com um maior detalhamento das identificações, as alterações na composição da comunidade poderiam ficar mais claras.

Os dados demonstraram que não houve diferença na abundância entre as trilhas estudadas, mas que a riqueza diferiu, com o BP apresentando maior número de táxons. Esta trilha apresenta menor volume de visitação por turistas do que a trilha PT. Com isso, a vegetação nos seus limites recebe menor impacto e, consequentemente, um maior número de recursos e de substratos permanece disponível, permitindo que aquelas espécies mais suscetíveis aos impactos provocados pela visitação sejam registradas (Haddad et al., 2001), como as ordens Colembolla e Diplura.

Quando comparadas as cotas altitudinais, não houve diferença significativa na abundância na trilha do Pico da Tijuca, o que também pode estar associado à maior prevalência de alguns grupos, como Orthoptera e Formicidae. A presença desses grupos em elevada abundância nas duas cotas altitudinais amostradas fez com que essas não apresentassem diferenças 

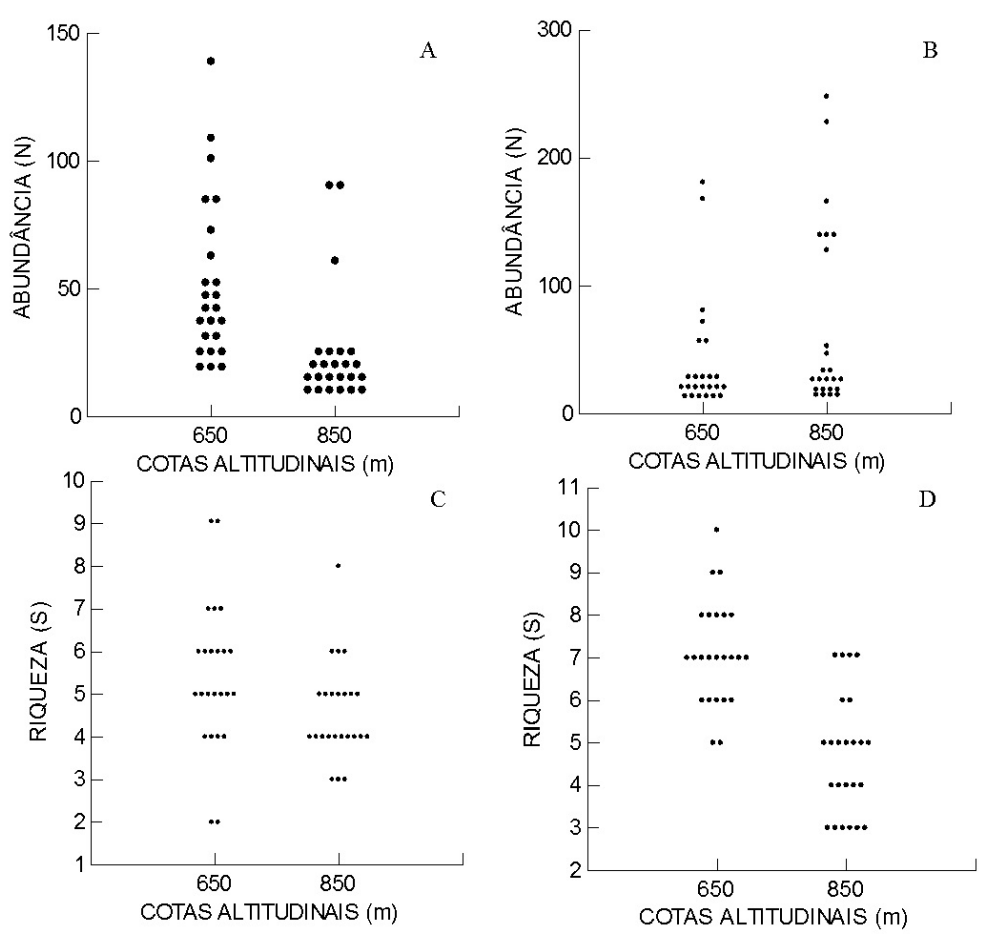

Figura 2. Abundância de indivíduos $(N)$ e riqueza de morfoespécies (S) da comunidade de artrópodes de serrapilheira amostradas nas altitudes $650 \mathrm{~m}$ e $850 \mathrm{~m}$ anm nas trilhas do Pico da Tijuca (A e C) e do Bico do Papagaio (B e D), Parque Nacional da Tijuca, Rio de Janeiro, Brasil.

Figure 2. Individual abundance $(\mathrm{N})$ and species richeness $(\mathrm{S})$ of macroarthropods community sampled at $650 \mathrm{~m}$ and $850 \mathrm{~m}$ above the sea level (a.s.l.), at Pico da Tijuca (A e C) and Bico do Papagaio (B e D) trails, Tijuca National Park, Rio de Janeiro, Brazil.

significativas. No entanto, é possível que, ao serem amostrado um maior número de cotas possíveis diferenças evidenciem-se.

Já em termos de número de táxons, as cotas altitudinais diferiram, com a menor cota (650 $\mathrm{m}$ anm) apresentando maior número de táxons. Esse resultado é corroborado pela ordenação obtida através do NMDS, que também mostrou que a estrutura da comunidade diferiu entre cotas altitudinais. Diferenças na riqueza e composição de espécies entre cotas altitudinais são apontadas para diferentes grupos biológicos, inclusive para insetos (Janzen, 1973; Wolda, 1987; Hasegawa et al., 2006). Na área de estudo, as diferenças no número de táxons e na composição da comunidade podem ser uma resposta à estrutura da vegetação, que na cota mais baixa das duas trilhas $(650 \mathrm{~m})$ parece ser mais heterogênea (observação pessoal), possivelmente devido à degradação, que é maior nessa altitude. Essa maior heterogeneidade da vegetação poderia causar também uma maior heterogeneidade na serrapilheira, fazendo com que um maior número de táxons pudesse ser encontrado nas amostras (Kumssa et al., 2004; Apigian et al., 2005).

Não foram observadas diferenças na abundância e no número de taxa entre as classes de distâncias da trilha. Esse resultado pode ser efeito da pequena distância entre as amostras (5 m), que não seria suficiente para identificar possíveis diferenças na abundância. Por exemplo, Garay \& Nataf (1982) encontraram mudanças na abundância de microartrópodes de serrapilheira conforme o aumento da distância da fonte de impacto utilizando distâncias superiores a $15 \mathrm{~m}$ a partir da fonte.

\section{CONCLUSÕES}

Os resultados do estudo indicaram que as diferenças na riqueza e composição estão relacionadas à altitude, entre as quais o grau de perturbação é distinto. 

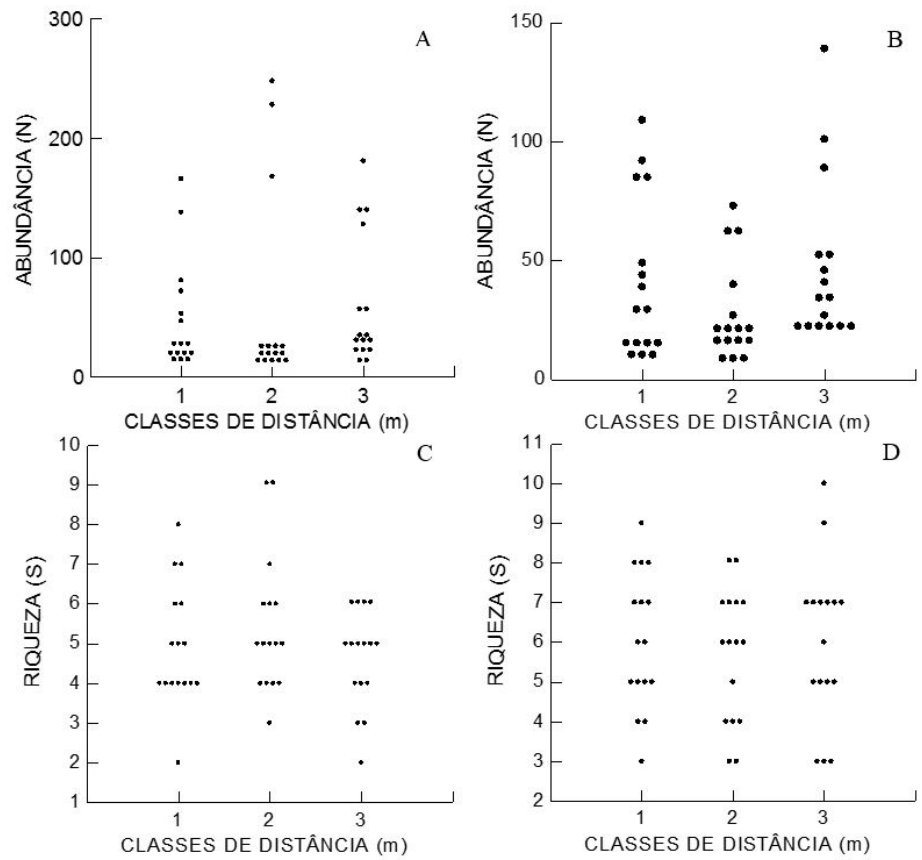

Figura 3. Abundância de indivíduos $(\mathrm{N})$ e riqueza de morfoespécies $(\mathrm{S})$ da comunidade de artrópodes de serrapilheira amostradas nas três classes de distância das trilhas do Pico da Tijuca (A e C) e do Bico do Papagaio (B e D), Parque Nacional da Tijuca, Rio de Janeiro, Brasil. $1=0,0 \mathrm{~m} ; 2=5,0 \mathrm{~m} ; 3=10,0 \mathrm{~m}$.

Figure 3. Individual abundance $(\mathrm{N})$ and species richeness $(\mathrm{S})$ of arthropods community sampled in three classes away from Pico da Tijuca (A e C) and Bico do Papagaio (B e D) trails, Tijuca National Park, Rio de Janeiro, Brazil. $1=0.0 \mathrm{~m} ; 2=5.0 \mathrm{~m} ; 3=10.0 \mathrm{~m}$.

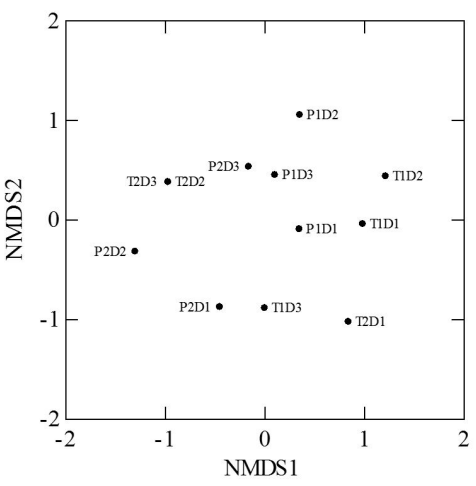

Figura 4. Ordenação das trilhas, cotas altitudinais $(\mathrm{P} 1$ = cota $650 \mathrm{~m}$ anm do Bico do Papagaio; P2 = cota $850 \mathrm{~m}$ anm no Bico do Papagaio; $\mathrm{T} 1=$ cota $650 \mathrm{~m}$ anm do Pico da Tijuca; T2 = cota $850 \mathrm{~m}$ anm do Pico d a Tijuca) e distâncias (D1 = 0 m; D2 = 5 m; D3 = $10 \mathrm{~m}$ ) em duas trilhas do Parque Nacional da Tijuca, Rio de Janeiro, Brasil.

Figure 4. Ordination of tracks, altitudes (P1 $=650 \mathrm{~m}$ asl of Pico do Papagaio; P2 = $850 \mathrm{~m}$ asl in Pico do Papagaio; $\mathrm{T} 1=650 \mathrm{~m}$ asl of Pico da Tijuca; T2 $=850 \mathrm{~m}$ asl of Pico da Tijuca) and distances (D1 $=0 \mathrm{~m}, 5 \mathrm{~m}=\mathrm{D} 2$, D3 $=10 \mathrm{~m}$ ) on two tracks of Parque Nacional da Tijuca, Rio de Janeiro, Brazil.
No entanto, para que essa hipótese seja confirmada, é necessário um maior esforço amostral, principalmente nas distâncias a partir da trilha e no número de cotas altitudinais. Somado a isso, um maior nível de refinamento na identificação dos grupos tornaria mais claras as respostas da comunidade de artrópodes de serrapilheira.

\section{AGRADECIMENTOS}

Os autores agradecem a T.C. Rocha-Pessôa e aos revisores pelas valiosas sugestões e correções. Agradecem também ao Parque Nacional da Tijuca pela autorização para realização das coletas do presente estudo.

\section{STATUS DA SUBMISSÃO}

Recebido: 2 jan., 2013

Aceito: 7 set., 2016 


\section{AUTOR(ES) PARA CORRESPONDÊNCIA}

\section{André Felippe Nunes-Freitas}

Laboratório de Ecologia Florestal e Biologia Vegetal, Departamento de Ciências Ambientais, Universidade Federal Rural do Rio de Janeiro UFRRJ, BR-465, Km 7, Seropédica, RJ, Brasil e-mail: afnfreitas@gmail.com

\section{REFERENNCIAS}

Alves ED Jr, Dias CAG. A cidade do Rio de Janeiro como equipamento de lazer: os esportes da natureza. In: Anais do Congresso Internacional Juego, Recreacion y Tiempo Libre; 2005; Montevidéu. Belo Horizonte: UFMG: CELAR; 2005.

Apigian KO, Dahlsten DL, Stephens SL. Fire and fire surrogate treatment effects on leaf litter arthropods in a western Sierra Nevada mixed-conifer forest. Forest Ecology and Management 2005; 221(1-3): 110-122. http://dx.doi. org/10.1016/j.foreco.2005.09.009.

Brasil. Sistema Nacional de Unidades de Conservação - SNUC. Lei no 9.985, de 18 de julho de 2000. Diário Oficial da República Federativa do Brasil, Brasília, DF (2000 jul 18).

Buzzi ZJ. Entomologia didática. 4. ed. Curitiba: Editora da UFPR; 2002.

Correia MEF, Oliveira LCM. Fauna de solo: aspectos gerais e metodológicos. Seropédica: Embrapa Agrobiologia; 2000.

Denlinger DL. Seazonal and annual avaliation of insect abundance in the Nairobi National Park, Kenya. Biotropica 1980; 12(2): 100-106. http://dx.doi.org/10.2307/2387725.

Freitas AVL, Leal IR, Uehara-Prado M, Ianuzzi L. Insetos como indicadores de conservação da paisagem. In: Rocha CFD, Bergallo HG, Sluys M Van, Alves MAS, organizadores. Biologia da conservação: essências. São Carlos: Editora RiMa; 2006a.

Freitas SR, Neves CL, Chernicharo P. Tijuca National Park: two pioneering restorationist initiatives in Atlantic Forest in Southeastern Brazil. Brazilian Journal of Biology 2006b; 66(4): 975-982. PMid:17299933. http://dx.doi. org/10.1590/S1519-69842006000600004.

Fundação SOS Mata Atlântica, Instituto Nacional de Pesquisas Espaciais - INPE. Evolução dos remanescentes florestais e ecossistemas associados do Domínio da Mata Atlântica no período 1985-1990. São Paulo: Fundação SOS Mata Atlântica, INPE; 1993.

Fundação SOS Mata Atlântica, Instituto Nacional de Pesquisas Espaciais - INPE. Atlas dos remanescentes florestais da Mata Atlântica e ecossistemas associados no período de 1995-2000. São Paulo: Fundação SOS Mata Atlântica: INPE; 2002. 44 p.
Garay I, Nataf L. Microarthropds as indicators of human trampling in suburban forests. In: Bornikamm R, Lee JA, Sebward MRD, organizadores. Urban ecology. London: Blackwell Scientific Publication; 1982. p. 201-207.

Haddad NM, Tilman D, Haarstad J, Ritchie M, Knops JMH. Contrasting effects of plant richness and composition on insect communities: a field experiment. American Naturalist 2001; 158(1): 17-35. PMid:18707312. http:// dx.doi.org/10.1086/320866.

Hafidi N, Garay I, Cancela da Fonseca JP. Colonization of Brown and bleached litter of a beech forest by edaphic macroarthropods. Pedobiologia 1998; 42(1): 23-32.

Hasegawa M, Ito MT, Kitayama K. Community structure of oribatid mites in relation to elevation and geology on the slope of Mount Kinanalu, Sabah, Malaysia. European Journal of Soil Biology 2006; 42: S191-S196. http://dx.doi. org/10.1016/j.ejsobi.2006.07.006.

Janzen DH. Sweep samples of tropical foliage insects: effects of seasons, vegetation types, elevation, time of day and insularity. Ecology 1973; 54(3): 687-708. http:// dx.doi.org/10.2307/1935359.

Kumssa DB, Aarde RJ Van, Wassenaar TD. The regeneration of soil micro-arthropod assemblages in a rehabilitating coastal dune forest at Richards Bay, South Africa. African Journal of Ecology 2004; 42(4): 346-354. http://dx.doi. org/10.1111/j.1365-2028.2004.00537.x.

Lecoq M. Gafanhotos do Brasil: natureza do problema e bibliografia. Montperllier: EMBRAPA/NMA: CIRAD/ PRIFAS; 1991.

Legendre P, Legendre L. Numerical ecology. Amsterdan: Elsevier Science BV; 1998.

Magurran AE. Ecological diversity and its measurement. Cambridge: University Press; 1988.

Masaki S, Walker TJ. Cricket life cycles. Evolutionary Biology 1987; 21: 349-423.

Matos DMS, Santos CJF, Chevalier DR. Fire and restoration of the largest urban forest of the world in Rio de Janeiro City, Brazil. Urban Ecosystems 2002; 6(3): 151-161. http:// dx.doi.org/10.1023/A:1026164427792.

McGeoch MAB. The selection, testing and application of terrestrial insects as bioindicators. Biological Reviews of the Cambridge Philosophical Society 1998; 73(2): 181-201. http://dx.doi.org/10.1017/S000632319700515X.

Mittermeier RA, Gil RP, Hoffman M, Pilgrim J, Brooks T, Mittermeier CG et al. Hotspots revisited: earth's biologically richest and most endangered terrestrial ecoregions. 2. ed. Boston: University of Chicago Press; 2005.

Myers N, Mittermeier RA, Mittermeier CG, Fonseca GA, Kents J. Biodiversity hotspots for conservation priorities. Nature 2000; 403(6772): 853-858. PMid:10706275. http:// dx.doi.org/10.1038/35002501. 
Oliveira-Filho AT, Fontes MA. Patterns of floristic differentiation among Atlantic Forest in Southeastern Brazil and the influence of climate. Biotropica 2000; 32(4b): 793-809. http://dx.doi.org/10.1111/j.1744-7429.2000. tb00619.x.

Pellens R, Garay I. A comunidade de macroartropodos edáficos em uma plantação de Coffea robusta Linden (Rubiaceae) e em uma floresta primária em Linhares, Espírito Santo, Brasil. Revista Brasileira de Zoologia 1999a; 16(1): 245-258. http://dx.doi.org/10.1590/S010181751999000100021 .

Pellens R, Garay I. Edaphic macroarthropod communities in fast-growing plantations of Eucalyptus grandis Hill ex Maid (Myrtaceae) and Acacia mangium Wild (Leguminosae) in Brazil. European Journal of Soil Biology 1999b; 35(2): 77-89. http://dx.doi.org/10.1016/S1164-5563(99)00209-5.

Rocha CFD, Bergallo HG, Alves MAS, Sluys M Van. A biodiversidade nos grandes remanescentes florestais do Estado do Rio de Janeiro e nas restingas da Mata Atlântica. São Carlos: RiMa Editora; 2003. 134 p.

Rosenzweig ML. Species diversity in space and time. Cambridge: Cambridge University Press; 2000. 460 p.

Silveira S No, Monteiro RC, Zucchi RA, Moraes RCB. Uso da análise faunística de insetos na avaliação de impacto ambiental. Scientia Agrícola 1995; 52(1): 9-15. http:// dx.doi.org/10.1590/S0103-90161995000100003.
Tanizaki-Fonseca K, Moulton TP. A fragmentação da Mata Atlântica no Estado do Rio de Janeiro e a perda da biodiversidade. In: Bergallo HG, Rocha CFD, Alves MAS, Sluys M Van, organizadores. A Fauna ameaçada de extinção do Estado do Rio de Janeiro. Rio de Janeiro: EDUERJ; 2000

Triplehorn CA, Johnson NF. Introdução ao estudo dos insetos. 7. ed. São Paulo: Editora Edgard Blücher; 2011. 816 p.

Wink C, Guedes JVC, Fagundes CK, Rovedder AP. Insetos edáficos como indicadores da qualidade ambiental. Revista de Ciências Agroveterinárias 2005; 4(1): 60-71.

Wolda H. Altitude, habitat and tropical insect diversity. Biological Journal of the Linnean Society 1987; 30(4): 313323. http://dx.doi.org/10.1111/j.1095-8312.1987.tb00305.x.

Wolda H. Fluctuation in abundance of some Homoptera in a neotropical forest. Geo-Eco-Trop 1977; 3: 229-257.

Wolda H. Fluctuation in abundance of tropical insects. American Naturalist 1978; 112(988): 1017-1045. http:// dx.doi.org/10.1086/283344.

Wolda H, Fisk FW. Seasonality of tropical insects II. Blattaria in Panama. Journal of Animal Ecology 1981; 50(3): 827-838. http://dx.doi.org/10.2307/4140.

Zacchi GP. Turismo ecológico e ecoturismo: diferenças e princípios éticos. Revista Diálogos \& Ciência 2004; 4: 1-16.

Zar JH. Biostatistical analysis. 4th. ed. New Jersey: PrenticeHall; 1999. 662 p. 